This article was downloaded by: [The University of Manchester Library] On: 27 J une 2013, At: 05:35

Publisher: Routledge

Informa Ltd Registered in England and Wales Registered Number: 1072954

Registered office: Mortimer House, 37-41 Mortimer Street, London W1T 3J H, UK

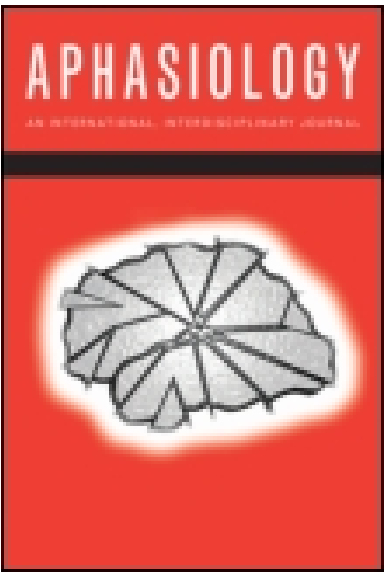

\title{
Aphasiology
}

Publication details, including instructions for authors and subscription information:

http:// www. tandfonline.com/loi/ paph20

\section{Domain-specific control of semantic cognition: A dissociation within patients with semantic working memory deficits}

\author{
Paul Hoffman ${ }^{a}$, Elizabeth J efferies ${ }^{b}$, Anthony Haffey ${ }^{b}$, \\ Thomas Littlejohns ${ }^{b} \&$ Matthew A. Lambon Ralph ${ }^{a}$ \\ ${ }^{a}$ Neuroscience and Aphasia Research Unit (NARU) , University \\ of Manchester, Manchester, UK \\ ${ }^{\mathrm{b}}$ Department of Psychology, University of York , York , UK \\ Published online: 01 Mar 2013.
}

To cite this article: Paul Hoffman, Elizabeth J efferies, Anthony Haffey , Thomas Littlejohns \& Matthew A. Lambon Ralph (2013): Domain-specific control of semantic cognition: A dissociation within patients with semantic working memory deficits, Aphasiology, 27:6, 740-764

To link to this article: http:// dx.doi.org/ 10.1080/ 02687038.2012.751578

\section{PLEASE SCROLL DOWN FOR ARTICLE}

Full terms and conditions of use: http://www.tandfonline.com/page/terms-andconditions

This article may be used for research, teaching, and private study purposes. Any substantial or systematic reproduction, redistribution, reselling, loan, sub-licensing, systematic supply, or distribution in any form to anyone is expressly forbidden.

The publisher does not give any warranty express or implied or make any representation that the contents will be complete or accurate or up to date. The accuracy of any instructions, formulae, and drug doses should be independently verified with primary sources. The publisher shall not be liable for any loss, actions, claims, proceedings, demand, or costs or damages whatsoever or howsoever caused arising directly or indirectly in connection with or arising out of the use of this material. 


\title{
Domain-specific control of semantic cognition: A dissociation within patients with semantic working memory deficits
}

\author{
Paul Hoffman ${ }^{1}$, Elizabeth Jefferies ${ }^{2}$, Anthony Haffey ${ }^{2}$, \\ Thomas Littlejohns ${ }^{2}$, and Matthew A. Lambon Ralph ${ }^{1}$ \\ ${ }^{1}$ Neuroscience and Aphasia Research Unit (NARU), University of Manchester, \\ Manchester, UK \\ ${ }^{2}$ Department of Psychology, University of York, York, UK
}

\begin{abstract}
Background: Semantic cognition depends on (a) semantic representations that code knowledge and (b) executive control processes that regulate access to this information such that relevant aspects are brought to the fore. It is not clear whether this second element, termed semantic control, draws on the same resources as executive processing in other domains.

Aims: We investigated the degree of independence between semantic control and domaingeneral executive function by studying non-semantic executive control in three patients who had semantic control deficits, and problems maintaining task-relevant conceptual knowledge in working memory.

Methods \& Procedures: Patients completed (a) a phonological working memory task that manipulated executive demands and (b) a series of working memory tasks that loaded a variety of executive functions: updating information, dividing attention, and manipulating the contents of working memory. These tasks featured digits and letters, placing minimal demands on semantic processing.

Outcomes \& Results: While two patients displayed clear executive deficits on these non-semantic tasks, the third case (patient JB) showed fully intact non-semantic control despite a clear control deficit for semantic tasks.

Conclusions: This dissociation suggests that executive control over semantic knowledge relies on partially distinct neural mechanisms to those involved in domain-general control.
\end{abstract}

Keywords: Semantic control; Semantic cognition; Short-term memory; Semantic aphasia; Executive function.

Although investigations of semantic memory have predominately focused on its representational structure (Mahon \& Caramazza, 2009; Martin, 2007; Patterson, Nestor, \& Rogers, 2007), in recent years there has been growing interest in the ways in which we access and manipulate these representations in the service of a particular goal or

Address correspondence to: Dr Paul Hoffman, Neuroscience and Aphasia Research Unit (NARU), Zochonis Building, School of Psychological Sciences, University of Manchester, Oxford Road, Manchester, M13 9PL, UK. E-mail: paul.hoffman@manchester.ac.uk

We are indebted to the patients and their carers for their generous assistance with this study. The research was supported by grants from the MRC (G0501632 \& MR/J004146/1) and NIMH (MH64445) and PH was supported by a studentship from the University of Manchester. 
task (Badre \& Wagner, 2002; Jefferies \& Lambon Ralph, 2006; Koenig \& Grossman, 2007; Thompson-Schill, D’Esposito, Aguirre, \& Farah, 1997). Appropriate responses to stimuli in our environment require not only that we store information about their properties, but also that we bring relevant aspects of this information to the fore when needed and prevent irrelevant aspects from intruding. This control is necessary because objects are associated with a vast quantity of information and only a small subset of this knowledge is helpful at any one time. Consider, for example, the two tasks of playing a piano versus moving a piano across a room (Saffran, 2000). While both involve the same object, a different subset of its properties is germane to each task (functions of the keys and pedals in the former case; its size, weight, value, and vulnerability to damage in the latter). Similar challenges arise in lexical-semantic processing. Many words have multiple meanings, and to comprehend them the appropriate meaning must be retrieved based on the current context (Rodd, Davis, \& Johnsrude, 2005). Even words that are not strictly polysemous can have meanings that vary in different situations (e.g., compare "phases of child development" with "phases of the moon"; Hoffman, Rogers, \& Lambon Ralph, 2011; Saffran, Bogyo, Schwartz, \& Marin, 1980). Moreover, in expressive tasks (even highly constrained ones like object naming) selection between multiple potential responses is required (e.g., the family pet could be referred to as "animal," "dog," "Alsatian," or "Fido"; see also Kan \& ThompsonSchill, 2004). In other situations aspects of an external stimulus must be ignored in order to focus on their deeper conceptual significance (e.g., the print on a newspaper is irrelevant when using it to swat a fly). Throughout this paper we will refer to these processes of activating contextually appropriate aspects of semantic knowledge and avoiding interference from irrelevant information as semantic control.

A fundamental issue in this research area is the following: What is the relationship between semantic control and other forms of executive control? To what extent are the neural systems that regulate semantic processing functionally independent from those that govern controlled processing in other domains, such as working memory and visual attention? A network of regions in dorsal prefrontal and inferior parietal cortex are implicated in controlled allocation of attention in a diverse range of tasks and have been termed "multiple demand" regions (Cristescu, Devlin, \& Nobre, 2006; Dosenbach, Fair, Cohen, Schlagger, \& Petersen, 2008; Duncan, 2006, 2010; Peers et al., 2005; Whitney, Kirk, O’Sullivan, Lambon Ralph, \& Jefferies, 2012). These regions are often activated for tasks that tap semantic control, suggesting that controlled processing in the semantic domain relies in part on this domainindependent system (Badre, Poldrack, Pare-Blagoev, Insler, \& Wagner, 2005; Collette, Hogge, Salmon, \& Van der Linden, 2006; Nagel, Schumacher, Goebel, \& D’Esposito, 2008; Nee, Wager, \& Jonides, 2007; Rodd et al., 2005; Thompson-Schill et al., 1997; Wagner, Pare-Blagoev, Clark, \& Poldrack, 2001). In contrast, other areas show a more selective response that is restricted to semantic control. For example, while the posterior portion of inferior prefrontal cortex (BA 44/9) responds to general cognitive control demands, the anterior aspects (BA 45/47) seem to be particularly important for semantic control (Gold \& Buckner, 2002; Gough, Nobre, \& Devlin, 2005; Nagel et al., 2008; Snyder, Feigenson, \& Thompson-Schill, 2007). In addition, posterior middle temporal gyrus is frequently activated in contrasts of high vs low semantic control but is not thought to be important for executive processing for other types of task (Bedny, McGill, \& Thompson-Schill, 2008; Gold et al., 2006; Noppeney, Phillips, \& Price, 2004; Rodd et al., 2005; Whitney, Jefferies, \& Kircher, 2011; Whitney, Kirk, O’Sullivan, Lambon Ralph, \& Jefferies, 2011). 
The emerging picture from these studies is that regulation of semantic knowledge depends on both domain-general multiple-demand regions and areas specialised for semantic control. It is not clear whether this partial neural specialisation can lead to a behavioural dissociation between semantic control and non-semantic executive control. In other words, are selective impairments of semantic control possible or is the semantic control network sufficiently integrated with the general executive system that it cannot be disrupted independently? Here we addressed this question by investigating executive control in multiple domains in patients with established semantic control deficits. In a series of studies we investigated patients with semantic control deficits following stroke (Jefferies \& Lambon Ralph, 2006; Noonan, Jefferies, Corbett, \& Lambon Ralph, 2010). Their profile, which we term "semantic aphasia" (SA), is characterised by multimodal semantic deficits that are highly sensitive to the cognitive control demands of the task being performed. For example, SA patients show poor comprehension of words with multiple meanings, in line with an inability to select from competing semantic representations. However, their performance improves when they are provided with contextual information that helps to resolve the ambiguity (Noonan et al., 2010). They also have difficulty inhibiting aspects of semantic knowledge that are irrelevant to the current task. Noonan et al. demonstrated this using a synonym matching task in which foils shared an irrelevant relationship with the probe (Samson, Connolly, \& Humphreys, 2007). Accuracy declined when the foil was strongly related to the target (e.g., they found it difficult to match piece with slice in the presence of the distractor word cake). In general, SA patients are particularly poor at accessing appropriate knowledge in unconstrained or open-ended tasks, in which a range of responses are possible, but their performance improves when the task strongly directs them towards a particular response (Corbett, Jefferies, \& Lambon Ralph, 2009, 2011; Hoffman, Jefferies, Ehsan, Hopper, \& Lambon Ralph, 2009; Jefferies, Patterson, \& Lambon Ralph, 2008; Soni et al., 2009).

In SA patients semantic control deficits are concomitant with executive control impairments on a range of non-semantic tasks and the severity of their general executive impairment is significantly correlated with the severity of their semantic deficit (Jefferies \& Lambon Ralph, 2006). One possibility is that these patients have a domain-independent control deficit that produces semantic and non-semantic deficits to similar degrees. However, since SA patients typically have large lesions affecting large swathes of frontal and/or temporoparietal cortex, it is also possible that multiple functional systems - some domain-general and some semantic-specific - are affected. In addition, SA patients sometimes show additional deficits in input or output processing (e.g., in speech production or visual perception) that can affect performance across a range of tasks, making interpretation of the correlations difficult.

Recently we have investigated three individuals who presented with a clear pattern of semantic control impairment but in a much "purer" form to that observed in SA (Hoffman et al., 2009; Hoffman, Jefferies, \& Lambon Ralph, 2011). These patients initially came to our attention because they had difficulty with verbal working memory tasks that required retention of semantic information, referred to as a semantic short-term memory (STM) deficit (Martin, Shelton, \& Yaffee, 1994). By directly comparing these patients to a case-series of SA patients we were able to demonstrate that their working memory problems were the result of a more general control problem for semantic information, similar in form to that seen in SA (Hoffman et al., 2009; Hoffman, Jefferies, et al., 2011). They showed the same effects of experimental manipulations of semantic control as SA patients, although the degree of impairment was 
much less severe. In fact they performed within the normal range on tests typically used to diagnose semantic impairment; it was only when tested with tasks designed to tax semantic control heavily that their deficits became apparent. We hypothesised that these patients suffered from a relatively pure semantic control deficit and that the semantic STM deficits observed as part of their profile arose because STM tasks tend to load heavily on executive control processes.

These three patients are unusual in the sense that they present with a clear semantic control deficit but have minimal perceptual and expressive difficulties. As such they are a particularly suitable group in which to address the relationship between semantic and domain-general executive control. In a previous study (Hoffman et al., 2009) we found some weak evidence suggestive of non-semantic control deficits in two of these patients. Their performance on a battery of executive control tests was at the lower end, or on some tests slightly outside, the normal range for age-matched controls. In the present study we tested in greater detail the status of domain-general control functions in this set of patients. Our aim was to determine whether their apparently circumscribed semantic control impairment was accompanied by more general executive deficits. To achieve this we employed more stringent tests of control taken from the working memory domain. Working memory tasks proved the most sensitive to semantic control deficits in this set of patients (Hoffman et al., 2009; Hoffman, Jefferies, et al., 2011). This may be because they have high intrinsic executive control demands, given that they involve activation and online maintenance of a number of items simultaneously (Baddeley, 1996; Engle, 2002; Miyake et al., 2000) (see also Jefferies, Hoffman, Jones, \& Lambon Ralph, 2008). We took two approaches to investigate non-semantic control. In Experiment 1 we varied executive demands in a phonological word detection task for which the meanings of the words were irrelevant. In Experiments 2-4 we employed digit and letter stimuli with minimal semantic content. Executive demands were changed by varying the need to update and manipulate the contents of working memory or to divide attention between two tasks. While we selected digits and letters for their lack of semantic associations, one aspect of letter processing, alphabetical ordering, does rely on stored knowledge. In Experiment 4 we made use of this fact to compare executive control relating to knowledge retrieval vs executive manipulation of new information within the same task.

\section{CASE DESCRIPTIONS}

The three patients tested have been described previously by Hoffman et al. (2009; Hoffman, Jefferies, et al., 2011). They were initially recruited on the basis that they showed semantic STM deficits, i.e., impaired verbal STM in tasks that emphasise semantic knowledge, with more preserved phonological STM. However, further testing (summarised in the next section) revealed that their STM deficits were symptomatic of a more general semantic control impairment which affected all sufficiently demanding tasks, irrespective of stimulus modality or whether short-term retention was involved.

JB was a 52-year-old man who left school at the age of 15 and was employed as a factory foreman. He suffered a left-hemisphere haemorrhagic CVA in April 2005. His language profile when assessed 4 months after the stroke was transcortical sensory aphasia. His speech was fluent and his ability to repeat verbal material was excellent but he displayed marked comprehension and word-finding difficulties. His language skills improved substantially after this initial period, such that at the time of the present study there were no obvious abnormalities in spontaneous speech or 


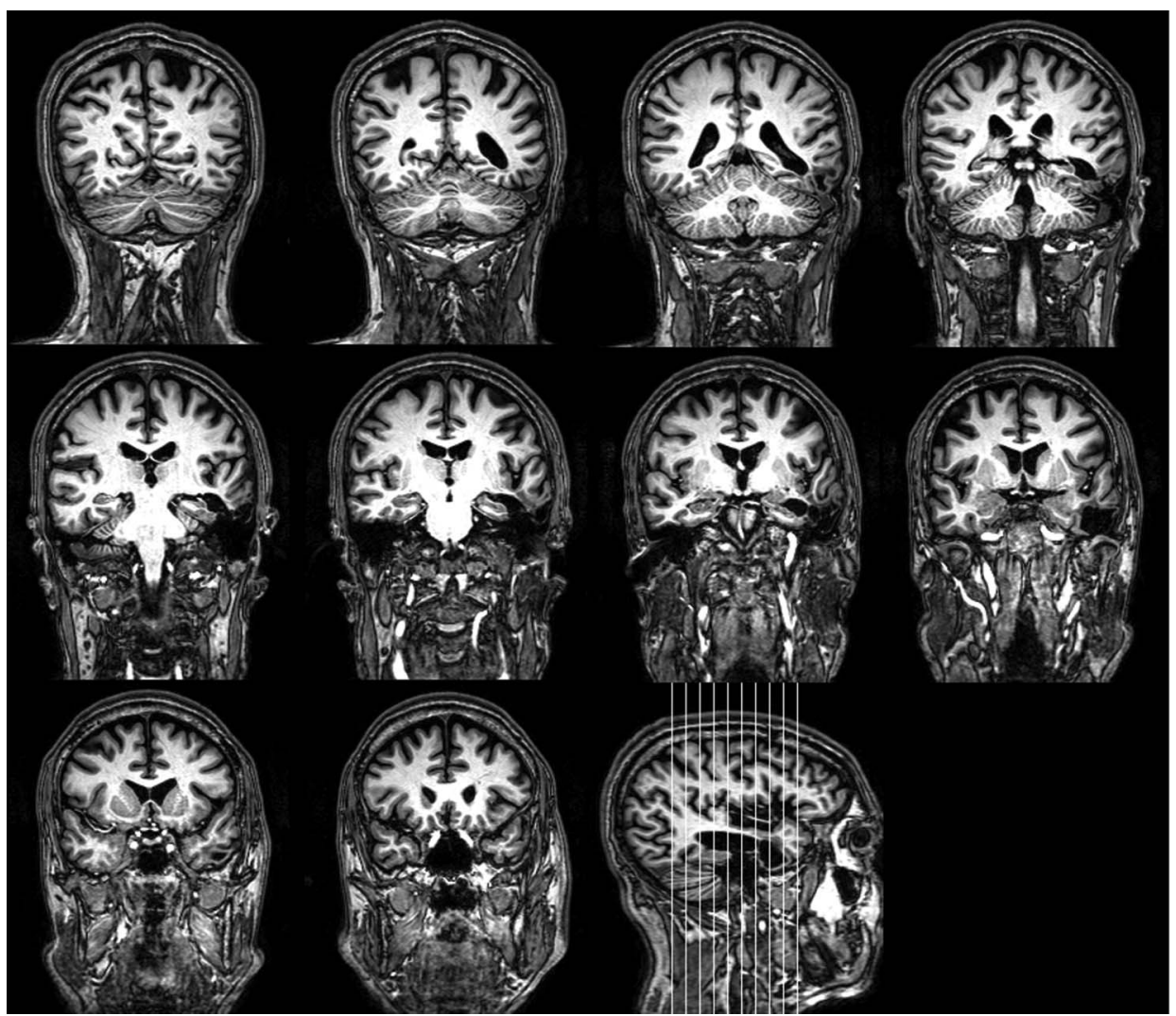

Figure 1. Coronal MRI slices for patient JB. Image is in radiological convention (left on right).

comprehension. A structural MRI scan was obtained, which revealed left hemisphere temporal damage (a series of coronal slices is shown in Figure 1). Loss of tissue was primarily along the fusiform and inferior temporal gyri (including the underlying white matter), and to a lesser extent the middle temporal gyrus. The superior temporal gyrus and sulcus were intact along their lengths.

ABU was a 54-year-old man who left school at the age of 15 and was employed in a local factory as a sheet metal worker until he experienced a CVA in June 2003. ABU initially presented with word-finding difficulty and mild comprehension problems, although his language abilities recovered after this initial period. When taking part in this study, his comprehension was good and his speech was fluent though punctuated by occasional hesitations and word-finding difficulties. His phrase length was slightly reduced. High-resolution structural imaging was not available for this patient. In a CT scan obtained shortly after the infarct, the left lateral ventricle appeared enlarged and the grey-white matter contrast in the basal ganglia was reduced on the left side, which could indicate a diffuse left-hemisphere partial infarction.

JHU was a 74-year-old man who left school aged 15 and spent most of his working life as an estate agent. He suffered a left hemisphere CVA in February 2008 and was referred to us in March 2009. We have no information on JHU's acute presentation; however when the present study began (15 months post-CVA) he presented with fluent 
speech with occasional word-finding difficulties. He displayed no obvious comprehension or speech production deficits. We were unable to obtain structural imaging for this patient.

\section{NEUROPSYCHOLOGICAL PROFILES}

The patients have completed a range of tests assessing semantic knowledge and control, STM, visuospatial skills and general executive control. These tests are summarised below; some of these results are reported in more detail by Hoffman et al. (2009; Hoffman, Jefferies, et al., 2011).

\section{Semantic processing and semantic control}

Patients completed a battery of standard semantic tests (the Cambridge Semantic Memory battery; Bozeat, Lambon Ralph, Patterson, Garrard, \& Hodges, 2000) as well as more targeted assessments that probed semantic control abilities (Noonan et al., 2010). Scores are shown in Table 1. The Cambridge Semantic Memory battery probes verbal and non-verbal semantic knowledge through tests of picture naming, spoken word-picture matching, and verbal and pictorial semantic association. Performance on these tests was largely within the normal range, indicating that these patients did not have marked deficits in semantic processing per se. In contrast, evidence for impairments did emerge on the tests that targeted semantic control more specifically. First, when asked to produce items belonging to particular semantic categories (e.g., animals), all patients performed poorly. This task has high semantic control demands because it is open-ended and patients must develop an appropriate retrieval strategy to generate exemplars. Second, patients were susceptible to the

TABLE 1

Tests of semantic processing and control

\begin{tabular}{|c|c|c|c|c|c|c|}
\hline & $\operatorname{Max}$ & $J B$ & $A B U$ & $J H U$ & Healthy control mean & Range \\
\hline \multicolumn{7}{|l|}{ General Semantics $^{\mathrm{a}}$} \\
\hline Naming & 64 & 58 & 63 & 61 & 62.3 & $57-64$ \\
\hline Word-picture matching & 64 & 64 & $61^{*}$ & 64 & 63.8 & $63-64$ \\
\hline \multicolumn{7}{|l|}{ Camel and Cactus test } \\
\hline Words & 64 & 62 & 57 & 59 & 60.7 & $56-63$ \\
\hline Pictures & 64 & 59 & 57 & $49^{*}$ & 59.1 & $51-62$ \\
\hline \multicolumn{7}{|l|}{ Semantic Control } \\
\hline Category fluency ( 8 categories) & - & $62^{*}$ & $58^{*}$ & $56^{*}$ & 121.5 & $75-162$ \\
\hline \multicolumn{7}{|l|}{ Single word comprehension } \\
\hline Appropriate cue & 60 & $58^{*}$ & $58^{*}$ & $58^{*}$ & 59.8 & $59-60$ \\
\hline Inappropriate cue & 60 & $48^{*}$ & $42^{*}$ & $51^{*}$ & 59 & $57-60$ \\
\hline \multicolumn{7}{|l|}{ Ignoring irrelevant distractors } \\
\hline Weak distractors & 42 & 41 & $28^{*}$ & $33^{*}$ & 41.5 & $41-42$ \\
\hline Strong distractors & 42 & 36 & $24^{*}$ & $24^{*}$ & 39.9 & $36-42$ \\
\hline \multicolumn{7}{|l|}{ Matching similar objects } \\
\hline Closely related & 64 & $61^{*}$ & $62^{*}$ & $63^{*}$ & 64 & $64-64$ \\
\hline Distantly related & 64 & $56^{*}$ & $47^{*}$ & $50^{*}$ & 62.6 & $59-64$ \\
\hline
\end{tabular}

*Abnormal scores.

${ }^{\mathrm{a}}$ Cambridge Semantic Memory Battery (Bozeat et al., 2000). 
following three manipulations of semantic control (described more fully in Hoffman, Jefferies, et al., 2011).

(1) The patients' comprehension of single words was close to normal when provided with a sentence that cued the meaning of the word. However, it was impaired when they had to overcome interference from a sentence that directed them towards an irrelevant aspect of the word's meaning (e.g., they found it hard to match ball with bat when accompanied by the sentence "She wore her new dress to the ball").

(2) They were impaired at detecting particular semantic relationships in the presence of irrelevant distracting information. In this task patients were asked to select the synonym for a particular word while ignoring a non-synonymous but semantically related word (e.g., matching piece with slice in the presence of the distractor word cake). Semantic control is needed to focus on the relevant relationship while inhibiting interference from the distractor word. This deficit was most pronounced when the irrelevant association was particularly strong.

(3) They were good at matching similar objects when the items shared a close semantic relationship (e.g., hat and cap) but performed more poorly when the items were more distantly related (e.g., hat and shoe). The trials involving distant semantic relationships required greater control because more controlled interrogation of the items' semantic representations was necessary to determine the connection between them.

These results indicate that while the patients showed little impairment in basic semantic processing, they were more impaired for tasks that demanded greater semantic control, either in terms of controlled processing of semantic knowledge or inhibition of irrelevant information.

\section{Verbal short-term memory}

Patients completed a number of verbal STM assessments, summarised in Table 2 and described in more detail by Hoffman et al. (2009). Their digit spans and ability to repeat lists of nonwords were intact, indicating preservation of phonological STM capacity. This suggests that any deficits in working memory tasks cannot be attributed to poor phonological span. Recall of word lists was impaired in ABU and JHU, suggesting impairment to semantically mediated STM. JB performed within the normal range on word list repetition, but this result must be interpreted in the context of his extremely good performance on the equivalent nonword test. Accordingly, the size of the "lexicality effect" (i.e., the recall advantage for words over nonwords by virtue of their lexical-semantic status) was significantly reduced in JB as well as in the other two patients. In addition, all three patients demonstrated a strong dissociation between semantic and phonological STM on probe detection tasks (Martin et al., 1994). These tasks require the detection of a word that shares either a phonological or semantic relationship with a probe. They performed normally on the phonological task but were impaired on the semantic version. In addition to its short-term maintenance demands, we have argued that this task has a strong semantic control requirement (Hoffman et al., 2009). 
TABLE 2

Tests of short-term memory and visuospatial skills

\begin{tabular}{|c|c|c|c|c|c|c|}
\hline & $\operatorname{Max}$ & $J B$ & $A B U$ & $J H U$ & Healthy control mean & Range \\
\hline \multicolumn{7}{|l|}{ Short-term memory ${ }^{\mathrm{a}}$} \\
\hline \multicolumn{7}{|l|}{ Digit span } \\
\hline Forwards & 8 & 7 & 5 & 7 & 6.8 & $5-8$ \\
\hline Backwards & 8 & 4 & 4 & 3 & 4.7 & $3-7$ \\
\hline Nonword lists & $\%$ & 57 & 32 & 29 & 28.9 & $18-46$ \\
\hline Word lists & $\%$ & 80 & $50^{*}$ & $40^{*}$ & 69.4 & $58-87$ \\
\hline Lexicality effect & $\%$ & $23^{*}$ & $18^{*}$ & $11^{*}$ & 40.6 & $34-51$ \\
\hline Rhyme judgement span & 9 & 8 & 6 & 9 & 6.98 & $4.7-9$ \\
\hline Category judgement span & 7 & $2.7^{*}$ & $2.7^{*}$ & $2^{*}$ & 6.15 & $4.7-7$ \\
\hline \multicolumn{7}{|l|}{ Visuospatial skills } \\
\hline \multicolumn{7}{|l|}{$\operatorname{VOSP}^{\mathrm{b}}$} \\
\hline Incomplete letters & 20 & 20 & 19 & 19 & 18.8 & $16-20$ \\
\hline Number location & 10 & 10 & 10 & 9 & 9.4 & $7-10$ \\
\hline Cube analysis & 10 & 10 & 9 & 10 & 9.2 & $6-10$ \\
\hline Rey figure copy & 36 & 33 & $29^{*}$ & NT & 34 & $30-36$ \\
\hline
\end{tabular}

*Abnormal scores. NT $=$ not tested.

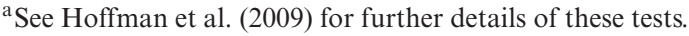

${ }^{\mathrm{b}}$ Visual Object and Space Perception Battery (Warrington \& James, 1991).

\section{Visuospatial skills}

Table 2 also shows the patients' scores on several components of the VOSP (Visual Object and Space Perception Battery; Warrington \& James, 1991) and their direct copy of the Rey complex figure. These scores were all normal with the exception of ABU's Rey copy, which fell very slightly outside the normal range. These findings indicate that any deficits on visually mediated executive tasks are unlikely to be a result of basic perceptual impairment.

\section{Executive function}

We administered a battery of standardised tests designed to probe executive control functions. This consisted of Raven's Standard Progressive Matrices (Raven, 1992), the Trail-making test (Tombaugh, 2004), the Elevator Counting test from the Test of Everyday Attention (Robertson, Ward, Ridgeway, \& Nimmo-Smith, 1994), the Stroop test, the Wisconsin Card-Sorting Test (Stuss et al., 2000), the Hayling and Brixton tests (Burgess \& Shallice, 1997), and the Biber Cognitive Estimation test (Bullard et al., 2004). Table 3 shows results on these tests alongside published norms from healthy age-matched controls. There were no consistent signs of executive impairment on these tests. All the patients were within the normal range of the Raven's non-verbal reasoning test, were able to complete the Elevator Counting task at a reasonable level while ignoring distracting information and showed normal interference effects on the Stroop test. However, JHU and ABU performed poorly on the executively demanding portion of the Trail-making test (Part B, which involves connecting letters and numbers in an alternating sequence) and $\mathrm{ABU}$ was impaired on the Wisconsin card-sorting task: his category score of 3 was below the tenth percentile and he took an abnormal number of trials to acquire the first category. 
TABLE 3

Tests of executive control

\begin{tabular}{|c|c|c|c|c|c|}
\hline Test & $J B$ & $A B U$ & $J H U$ & $\begin{array}{c}\text { Healthy } \\
\text { control mean }\end{array}$ & Impaired cut-off \\
\hline Raven Standard Progressive Matrices/60 & 36 & 34 & 36 & 50 & $<27$ \\
\hline \multicolumn{6}{|l|}{ Trails Test } \\
\hline Part A completion time & 51 & 42 & 42 & 32 & $>51$ \\
\hline Part B completion time & 75 & $180^{*}$ & $100^{*}$ & 64 & $>93$ \\
\hline \multicolumn{6}{|l|}{ TEA Elevator counting } \\
\hline No distraction/7 & 7 & 7 & 7 & 6.6 & $<6$ \\
\hline With distraction/10 & 10 & 5 & 8 & 8.2 & $<4$ \\
\hline \multicolumn{6}{|l|}{ Stroop test } \\
\hline Word reading & 88 & $63^{*}$ & 72 & 108 & $<68$ \\
\hline Colour naming & 94 & 56 & $49^{*}$ & 80 & $<50$ \\
\hline Conflict condition & 39 & 26 & 39 & 45 & $<25$ \\
\hline Interference effect & -6 & -4 & 11 & 0 & $<-20$ \\
\hline \multicolumn{6}{|l|}{ Wisconsin Card-Sorting Test } \\
\hline Categories achieved/6 & 6 & 3 & 4 & & \\
\hline Category \%ile range & $>16$ th & $6-10$ th & $>16$ th & & \\
\hline Errors made/128 & 15 & 50 & 57 & 27 & $>64$ \\
\hline Trials to first category & 12 & $30^{*}$ & 10 & & $>19$ \\
\hline Brixton Test of Spatial Anticipation/54 & 41 & 30 & 32 & 37 & $<29$ \\
\hline \multicolumn{6}{|l|}{ Hayling test } \\
\hline Section A errors & 0 & 1 & 0 & & \\
\hline Section B errors & $40^{*}$ & 1 & 3 & 10 & $>30$ \\
\hline Biber Cognitive Estimation test/20 & $12^{*}$ & $14^{*}$ & 15 & 18.1 & $<15$ \\
\hline
\end{tabular}

*Abnormal scores. Stroop scores age-adjusted. TEA = Test of everyday attention (Robertson et al., 1994).

JB was impaired on two tasks: the Hayling test and the Cognitive Estimation task. These tasks are notable because they both test executive regulation of semantic information. The Hayling test requires patients to complete sentences. In Part A they are asked to supply an appropriate word to complete each sentence and in Part B to complete each sentence with a word that is unrelated to the sentence. JB made no errors on Part A, indicating intact comprehension and word retrieval, but was impaired on Part B, where executive control is needed to inhibit semantic information relating to the sentence and instead generate a novel response. In the Cognitive Estimation task patients are asked to estimate quantities relating to familiar items that they would not be expected to know precisely (e.g., How many slices of bread are there in an average loaf?). This requires executive regulation to probe semantic knowledge in a flexible way and thereby generate a reasonable estimate. JB and ABU were both impaired on this test, with JHU also performing somewhat poorly.

\section{Summary}

The neuropsychological profiles of the three patients indicated deficits of semantic control combined with preserved visuospatial skills and preservation of phonological STM capacity (i.e., memory for digits and nonwords). In terms of executive function no patient was consistently impaired on all of these tasks, although ABU and JHU displayed weakness on some tests, which might indicate a mild general executive deficit. 
JB's profile was particularly interesting: he failed only those tasks involving executive regulation in the semantic domain, suggesting that his control deficit might be specific to semantic knowledge. In the remainder of the study we investigated this possibility in more detail using a number of working memory tests designed to be sensitive to mild non-semantic executive deficits.

\section{EXPERIMENT 1: STRONG VS WEAK RELATIONSHIPS IN PHONOLOGICAL STM}

In the first experiment we sought evidence for sensitivity to executive demands in phonological processing. A number of studies have manipulated executive demands on semantic processing by varying the strength of the semantic relationship between two words (Badre et al., 2005; Noonan et al., 2010; Wagner et al., 2001; Whitney, Kirk, et al., 2011). Likewise the three patients described here were impaired in their ability to detect semantic relationships between distantly related items, particularly in the context of a demanding STM task (see Table 1 and Hoffman, Jefferies, et al., 2011). By analogy, we reasoned that the detection of weak phonological relationships would be more executively demanding than detecting items with greater phonological overlap. We designed a task based on the rhyming judgement task (Martin et al., 1994), in which patients are presented with a word list and a probe and decide whether the probe rhymes with any of the list words. Background testing indicated that all three patients performed normally on this task (see Table 2). However, it is likely that rhyme detection has low executive demands because rhyming words are phonologically very similar and the overlapping rime unit is highly dominant in English (Treiman \& Danis, 1988; Treiman \& Kessler, 1995): for example, young children are better at segmenting words and detecting phonological similarity at the rime level than at the single phoneme level (Ziegler \& Goswami, 2005). We contrasted rhyme detection with a more demanding phonological detection task in which the matching words shared only a single phoneme. We reasoned that detecting weaker phonological relationships is likely to involve greater controlled processing and therefore higher executive demands (in a similar fashion to weak semantic relationships). If the patients had domain-general executive deficits, we would expect to observe poorer performance on the single phoneme task relative to rhyme detection. However, neither task was dependent on semantic processing of the words, so a control deficit specific to semantics would not lead to impairment on either task.

\section{Method}

Materials. Patients completed two phonological judgement tasks in which they were presented with an auditory list of CVC words and asked to decide whether a probe word was phonologically related to any of them (see Table 4 for examples). For the rhyming task, they were asked to respond "yes" if the probe rhymed with any of the words from the list.

For the phoneme task they were asked to decide whether the probe contained any of the same phonemes as those in any of the words in the list. A match was present on half of the trials. The phoneme task was more difficult than the rhyme task in a number of ways: 
TABLE 4

Example trials in Experiment 1

\begin{tabular}{llll}
\hline Condition & \multicolumn{1}{c}{ List } & Probe & Correct response \\
\hline Rhyme & dame, vile, note & trial & Yes \\
Rhyme & fog, limb, cage & pool & No \\
Phoneme & buzz, cod, wheat & wedge & Yes $($ shares $<\mathrm{w}>$ ) \\
Phoneme & bait, pad, mark & lord & Yes $($ shares $<\mathrm{d}>$ ) \\
Phoneme & guess, nape, hush & seat & Yes $($ shares $<\mathrm{s}>$ ) \\
Phoneme & curve, pug, ten & soul & No \\
\hline
\end{tabular}

The matching word on "yes" trials is shown in bold.

(1) The matching word, when it was present, only shared a single phoneme with the probe, whereas rhyming words always shared two phonemes. This meant that the phonological relationship was weaker and less salient, and more detailed processing was needed to confirm the presence of a match.

(2) The shared phoneme could appear either at the start of the end of the word, whereas the overlap between the rhyming words was entirely predictable (i.e., always occurred at the end of the word). This meant that, in the shared phoneme condition, the patients had to direct attention to all elements of each word to decide whether a match was present.

(3) The shared phoneme could appear in different places in the two words (e.g., bat and cub). This manipulation meant that participants were required to identify two potential "probe phonemes" and then compare both of these with the list of potential targets, substantially increasing the requirement to maintain and manipulate phonological information in working memory.

Word frequency, biphone frequency, and neighbourhood density for the words used in each test are presented in Table 5. Mean values for probes, their matching targets and the non-matching filler words are reported separately. $T$-tests indicated that the rhyme and phoneme tasks did not differ significantly for any of these values $(t<1.8$, $p>.05)$.

TABLE 5

Psycholinguistic properties of words for Experiment 1

\begin{tabular}{llcr}
\hline Property & Words & Phoneme test & Rhyme test \\
\hline Log word frequency $^{\mathrm{a}}$ & Probes & $1.25(0.71)$ & $1.25(0.75)$ \\
& Targets & $1.44(0.73)$ & $1.29(0.67)$ \\
& Fillers & $1.36(0.77)$ & $1.39(0.77)$ \\
Biphone frequency $^{\mathrm{b}}$ & Probes & $869(1266)$ & $768(1008)$ \\
& Targets & $825(825)$ & $821(1047)$ \\
& Fillers & $1084(1582)$ & $1084(1586)$ \\
Phonological neighbourhood density $^{\mathrm{c}}$ & Probes & $1.19(0.26)$ & $1.26(0.28)$ \\
& Targets & $1.20(0.28)$ & $1.20(0.26)$ \\
& Fillers & $1.21(0.26)$ & $1.21(0.26)$ \\
\hline
\end{tabular}

Standard deviations are shown in parentheses.

${ }^{\mathrm{a}}$ From CELEX (Baayen, Piepenbrock, \& van Rijn, 1993). ${ }^{\mathrm{b}}$ Obtained using N-watch (Davis, 2005).

${ }^{\mathrm{c}}$ Levenshtein distance (Yarkoni, Balota, \& Yap, 2008). 
Procedure. Rhyme and phoneme tasks were completed on different days, following the same procedure. The task was first explained using a number of examples of matching and non-matching words. Patients were specifically instructed to pay attention to the sounds of the words, rather than how they were spelled. They were then presented with 24 pairs of words, read aloud by the experimenter, and asked whether each pair matched. This tested whether they understood the nature of the phonological judgements in each condition. Following this, they were presented with lists of 2, 3, 4, and 5 words, with 24 lists at each length. Lists were read out at a rate of one word per second and were followed immediately by the probe. The same lists were presented in the rhyming and phoneme conditions, but with different probes.

Control participants. A total of 16 healthy controls were recruited from the volunteer panel of the University of York. They had a mean age of 71 years $(S D=4.4$; range $=62-78)$ and had completed a mean of 12.3 years in formal education $(S D=$ 2.0 ; range $=10-17$ ). None had any history of neurological illness.

Data analysis. The control participants were well matched in age to patient JHU but were somewhat older than JB and ABU. They also tended to have spent more time in formal education than the patients. To determine the degree to which these demographic factors could influence performance, we first computed the correlations of age and educational level with performance in each of the four conditions in the experiment. We then proceeded to compare each of the patients to the control group. In all experiments impairment in a particular condition was inferred if there was a significant difference between the patient score and the controls, using Crawford and Howell's (1998) modified $t$-test (one-tailed $p<.05$ ). This is denoted in all figures with an asterisk. We also used the Revised Standardised Difference Test (RSTD; Crawford \& Garthwaite, 2005) to determine whether patients showed an abnormally large dissociation between two conditions. This test indicates whether the difference between two conditions exhibited by a patient is significantly larger than that observed within the control group.

\section{Results}

Effects of age and educational level in control participants. Performance was not significantly correlated with age $(|r|<0.23, p>.4)$ or with educational level $(|r|<0.36$, $p>.17)$ in any of the conditions, suggesting that these factors are unlikely to strongly influence performance in the experiment. Therefore it was possible to compare each of our patients to the control group, despite them being slightly older and more highly educated than JB and ABU.

Evidence for impairment in the patients. Figure 2 shows correct responses to the word pairs and the word lists in each condition. None of the patients was impaired at judgements on word pairs, indicating that they understood the nature of the task and were capable of making both types of judgement accurately. However, evidence of impairment was found when the same judgements were made for longer lists of words. $\mathrm{ABU}$ and JHU were both marginally impaired when making the more demanding single phoneme judgements at the word list level (modified $t$-test: $p=.06$ in each case). No impairment was observed when the less-demanding rhyme judgements were made to word lists. RSDT was used to assess whether a dissociation was present between 


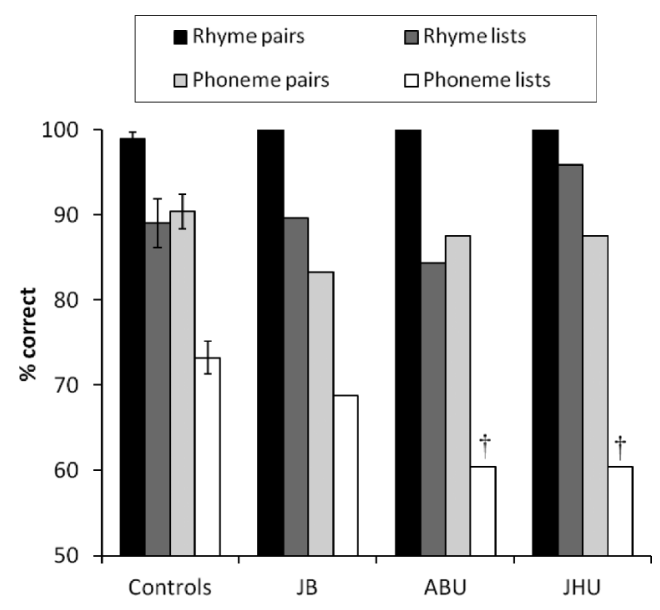

Figure 2. Phonological STM Tasks. †Marginally impaired performance (modified $t$-test; $p=.06$ ).

rhyme judgements and single phoneme judgements for word lists. JHU showed such a dissociation, $t(15)=2.31, p=.018$, indicating that he was significantly more impaired for the single phoneme judgements than for the rhyme judgements (in fact, he scored slightly above the control mean in the rhyming condition). In contrast to the other patients, JB was unimpaired for both types of phonological judgement. He did not show a dissociation between rhyme judgements and single phoneme judgements.

\section{Discussion}

ABU and JHU were both impaired when asked to make executively demanding phonological judgements for lists of words. These deficits cannot be attributed to difficulty in comprehending the task or in basic phonological processing or segmentation, since both made the same judgements successfully to pairs of words. Background testing indicated that these patients do not have deficits to phonological storage per se (see Table 2). Instead their poor performance might have been a consequence of the additional executive demands of detecting words that share a weak phonological relationship. Alternatively, their deficits might indicate very mild phonological processing deficits, which are only detectable on the most demanding tasks. In any case, this experiment demonstrates that both ABU and JHU have STM deficits that extend beyond the semantic domain.

In contrast, JB displayed no impairment on this task, even for the most demanding phonological judgements. Since JB shows clear deficits when required to detect weak semantic relationships, this suggests that his control deficit may be specific to the semantic domain. However, this conclusion must be considered with caution as the control participants were somewhat older than JB. Although performance was not correlated with age in the control group, it remains possible that a younger control group would have performed at a higher level. To address this issue, in the remaining experiments we recruited younger control participants who were matched in age to JB and ABU, as well as older individuals of similar age to JHU. 


\section{EXPERIMENT 2: N-BACK TASK}

The n-back task is a working memory task commonly used to investigate executive function (e.g., Awh et al., 1996). Participants are presented with a single digit on each trial and are asked whether it is the same as the number presented $n$ trials previously. When $n=1$ this is relatively undemanding, as the participant simply has to compare the current digit to the one presented immediately before. As $n$ increases, the executive demands of the task increase because the participant is required to recall digits from earlier trials while retaining the intervening digits for use in later trials. In addition the participant has to continuously update the memory set, discarding old trials while ensuring that new digits are encoded in the correct order. Here, we used 2-back and 3 -back trials to assess non-semantic executive control in our patients, comparing these to the less demanding 1-back condition.

\section{Method}

Task. Patients completed three versions of a running span task, each involving auditory presentation of single digits between 1 and 9. Patients completed the 1-back condition first. On each trial the experimenter read aloud a single digit and the patient was asked whether it was the same or different to the digit read out on the previous trial. In the 2-back and 3-back conditions digits were presented singly as before but patients had to decide whether each digit was the same as the one presented either two or three trials previously. There was no time limit on responses and each condition consisted of 25 trials. Each condition was designed such that half of the trials required yes responses and half required no.

Control participants. A total of 16 healthy participants were recruited from the Manchester and York participant panels to take part in Experiments 2, 3, and 4. They were divided into two subgroups based on age, each containing eight participants. The younger control group were matched in age to JB and ABU: they had a mean age of $55(S D=6.5$; range $=45-62)$. The older control group were matched to JHU and had a mean age of $70(S D=4.7$; range $=66-78)$. Educational level was 14.8 years on average in the younger group $(S D=2.8$; range $=12-19)$ and 13.6 years in the older group $(S D=2.9$; range $=10-18)$.

Data analysis. The following procedures were adopted in Experiments 2, 3, and 4. To ensure that differences in age and educational level could not bias the results, we checked for correlations between these factors and task performance in each condition in the entire control group $(N=16)$. Following this we tested for evidence of impairment in each patient using modified $t$-tests. Tests were performed comparing each patient to the full group of 16 controls and to their own age-matched subgroup, with impairment only being assumed if both tests were significant. RSDT was used to assess the significance of dissociations between particular conditions. These tests were also performed against the full control group (denoted $t_{1}$ ) and the patient's age-matched subgroup (denoted $t_{2}$ ).

\section{Results}

Effects of age and education. Within the control group, there were no significant correlations between n-back performance and age $(|r|<0.31, p>.24)$ or with educational level $(|r|<0.08, p>.77)$, for any of the conditions. 


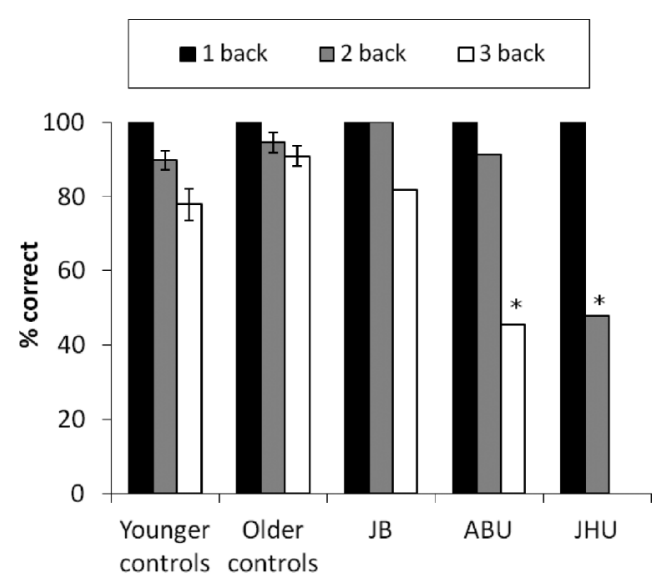

Figure 3. N-Back task. Asterisks denote significantly impaired performance (modified $t$-test; $p<.05$ ).

Evidence for impairment in the patients. Results are shown in Figure 3. While all of the patients performed at ceiling in the 1-back condition, there were signs of impairment on the more executively demanding conditions. JHU responded at chance in the 2-back condition and could not attempt the 3-back condition. ABU was able to complete the 2-back condition successfully but dropped to chance in the 3-back condition. In contrast, JB's performance was comparable to that of controls in all three conditions. RSDT confirmed that ABU showed a significantly larger difference between the 2-back and 3-back conditions than was observed in the controls $\left(t_{1}=2.61, p=.01\right.$; $\left.t_{2}=1.95, p=.05\right)$. JB showed no such interaction $\left(t_{1}=1.05, p=.15 ; t_{2}=0.75\right.$, $p=.24)$. The statistical test could not be performed for JHU as he did not complete the 3-back condition. However, he did exhibit a striking dissociation between $100 \%$ accuracy in the 1-back condition and chance performance in the 2-back condition.

\section{Discussion}

The n-back task revealed executive impairment in ABU and JHU. These deficits are unlikely to be a result of reduced phonological storage capacity, as both patients displayed preserved digit span and repetition of nonword lists in background testing. In contrast, JB showed no evidence of executive impairment, again indicating a dissociation between semantic and non-semantic control in his case.

\section{EXPERIMENT 3: COMPLEX MEMORY SPAN}

The ability to update representations in working memory and shift attention between two tasks are thought to be key aspects of executive function (Miyake et al., 2000). In this experiment we tested these abilities using two "complex span" tasks that required patients to encode and retain a series of stimuli while simultaneously completing an unrelated task (Unsworth, Heitz, Schrock, \& Engle, 2005). As in the previous experiment we focused on retention of stimuli that have little intrinsic semantic significance: numbers and letters. In the Counting Span task patients attempted to recall digits while completing a "distracting" visual search task. They were presented with a visual display and asked to count how many of a particular type of shape were present 
and remember this total. After seeing a series of such displays they were asked to recall the totals from each display. Thus, in this task both the STM component and the distracting material were non-semantic. In the Reading Span task patients were presented with a series of sentences and decided whether each one made sense. Each sentence was followed by a letter of the alphabet that they were instructed to remember. After seeing a series of sentence-letter pairs, they tried to recall the letters. This test examined the effect on irrelevant semantic processing on the retention on non-semantic verbal information in STM.

\section{Method}

We used the verbal response versions of the complex span tasks developed by Unsworth et al. (2005), the code for which is available on the following website: http:// psychology.gatech.edu/renglelab/Eprime1.html. They were presented using a laptop computer running Eprime software.

Counting span. On each trial patients saw a series of visual displays consisting of squares and circles in two colours (light and dark blue) on a grey background. For each display they were asked to count the number of dark blue circles present in the display, and to say aloud and remember this total. A number of such displays were presented sequentially, followed by a cue to recall. Upon seeing the cue the participant attempted to recall the totals for all the displays they had just seen, in the order in which they were presented. Following recall the next trial began with a new series of displays. There were between two and six displays on each trial and there were 15 trials in total. There was no time limit placed on responses.

Reading span. On each trial participants saw a series of written sentences, each followed by a single upper-case letter. They were asked to each the sentence aloud and decide whether it was meaningful. Half of the sentences were meaningful and half contained an inappropriate word (e.g., "Raising children requires a lot of dust and the ability to be firm"). After making the sentence decision participants were asked to read the letter aloud and attempt to remember it. After they had done so, the display was updated to show a new sentence and letter. This process was repeated until a cue to recall was presented, at which point the patient attempted to recall the letters in the order in which they were presented. Each trial consisted of between two and five sentences/letters, for a total of 12 trials.

For both tasks the proportion of letters or digits correctly recalled on each trial was calculated. We also recorded performance on the distracting tasks.

Control participants. The 16 participants from Experiment 2 took part in this experiment.

\section{Results}

Effects of age and education. Within the control group there were no significant correlations between performance and age $(|r|<0.12, p>.65)$ or with educational level $(|r|<0.40, p>$.12), for either task. 


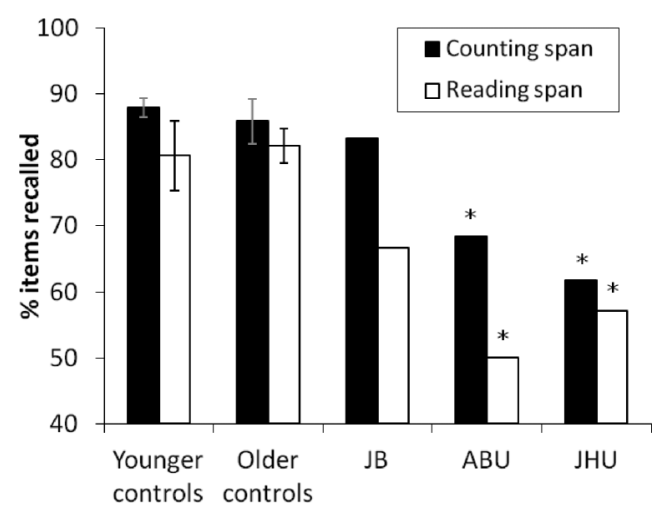

Figure 4. Counting and reading span results. Asterisks indicate significantly impaired performance (modified $t$-test; $p<.05)$.

Evidence for impairment in the patients. Results for patients and healthy controls are shown in Figure 4. ABU and JHU were impaired on significantly impaired on both tasks but JB was not significantly impaired on either. RSDT indicated that there was no dissociation between the two tasks in $\mathrm{JB}\left(t_{1}=0.60, p=.28 ; t_{2}=0.12, p=.46\right)$ or $\mathrm{JHU}\left(t_{1}=0.97, p=.17 ; t_{2}=0.69, p=.26\right)$. A significant dissociation was present in $\mathrm{ABU}$, although only when he was compared to the younger control subgroup $\left(t_{1}=\right.$ $\left.0.18, p=.43 ; t_{2}=1.92, p=.048\right)$. Although ABU was impaired on both tasks, this result suggests that his impairment may have been more severe on the counting span task.

Performance on the distracting tasks was good in all cases. All patients correctly counted the shapes on at least $85 \%$ of occasions $(\mathrm{JB}=95 \%$; $\mathrm{ABU}=85 \%$; JHU $=$ $95 \%$; control mean $=96 \%$ ) and responded correctly to over $90 \%$ of the sentences $(\mathrm{JB}=98 \%$; ABU $=93 \%$; JHU $=95 \%$; control mean $=99 \%)$.

\section{Discussion}

This experiment provided further evidence for general executive deficits in ABU and JHU. Both showed impaired letter and digit recall under executively demanding conditions. In contrast, background testing indicated that when tested without distracting concurrent tasks these patients had digit spans within the normal range. Again, JB showed no evidence of executive impairment when recalling materials with minimal semantic content, suggesting a dissociation between semantic control and general executive function in his case. It is worth noting that, although the sentence judgements were based on semantic knowledge, they were very easy and the yes/no response format was likely to minimise any semantic control requirement. In fact, JB performed at the lower end of the control range on this task, perhaps indicating a small contribution of semantic control.

\section{EXPERIMENT 4: MANIPULATION VS STORAGE OF LETTER LISTS}

Processing and manipulation of items in working memory is thought to be more executively demanding than passive storage (Belleville, Rouleau, \& Caza, 1998; Collette 
et al., 1999; Engle, Tuholski, Laughlin, \& Conway, 1999). In the final experiment we varied executive demands by asking participants to manipulate letter sequences held in working memory in different ways. We again used letters as they have little intrinsic semantic content. In the first, low-control, condition patients were asked to recall letter strings exactly as presented. This verbatim repetition required no manipulation or processing of the letters, so was not expected to strongly engage executive processes. In the remaining two conditions patients were asked to manipulate the order of the letters before recalling them, increasing executive demands. In a backward recall condition patients were asked to reverse the order of the letter string. This required additional processing of the contents of working memory but there was no need to access stored knowledge. In a final, alphabetical, condition patients were asked to reproduce the letters in alphabetical order (Belleville et al., 1998). This condition required participants to use their acquired knowledge of the alphabet to regulate their response. It is not clear whether knowledge of the alphabet is supported by semantic memory or whether it relies on other cognitive systems (for example, since the alphabet is a welllearned auditory sequence, it may rely on phonological coding). However, we included this condition because, in common with semantic control tasks, it requires manipulation of items held in working memory based on stored long-term knowledge of their properties.

\section{Method}

Task. Participants were presented with auditory sequences of letters and attempted to recall them in one of three ways. In the forward recall condition they reproduced the string of letters exactly as presented. In the backward recall condition they were asked to recall the letters in the reverse order to that presented. In the alphabetical condition they attempted to recall the letters in alphabetical order. These three conditions were interspersed, with a verbal cue on each trial indicating how to recall the letters ("forward," "backward," or "alphabetical"). The timing of the cue varied: on half of the trials it was given prior to presentation of the letters and on the remaining half immediately after. Recall began immediately after presentation of the cue and letter string. All patients received strings containing five letters, for a total of 48 trials. However, ABU performed somewhat poorly on these even for forward recall, so was also tested with 48 lists of four letters. The proportion of letters recalled in the correct sequence was used as a measure of accuracy.

To test knowledge of the alphabet, patients also completed a two-alternative forced choice alphabetic ordering task. On each trial, patients were visually presented with two letters and asked which came first in the alphabet. There were 28 trials.

Control participants. Of the 16 participants from Experiments 2 and 3, 15 also took part in this experiment. One participant from the older subgroup was unavailable. All control participants completed the five-letter lists. Seven of the younger controls also completed the four-letter lists, providing a matched control group for ABU.

\section{Results}

Effects of age and education. Within the control group there were no significant correlations between performance and age $(|r|<0.22, p>.41)$ or with educational level $(|r|<0.17, p>.53)$, for forward, backward, or alphabetical recall. 


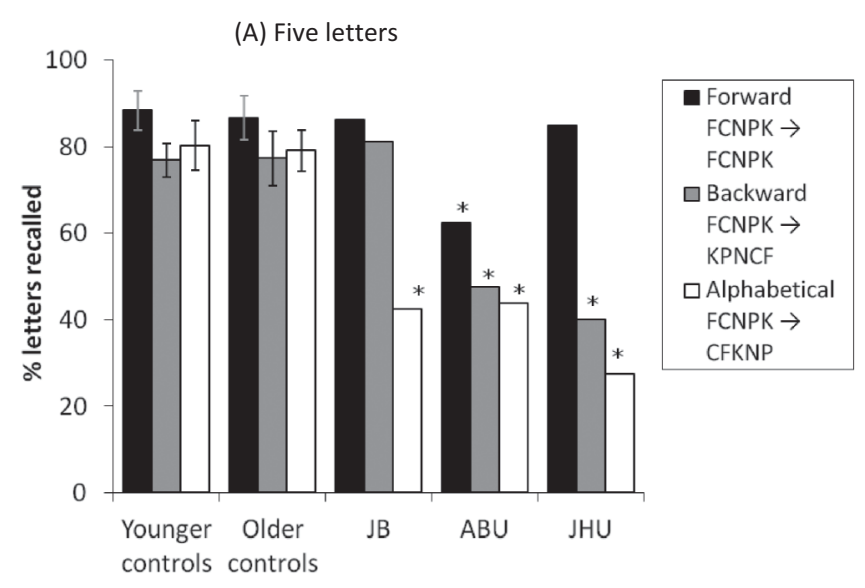

(B) Four letters

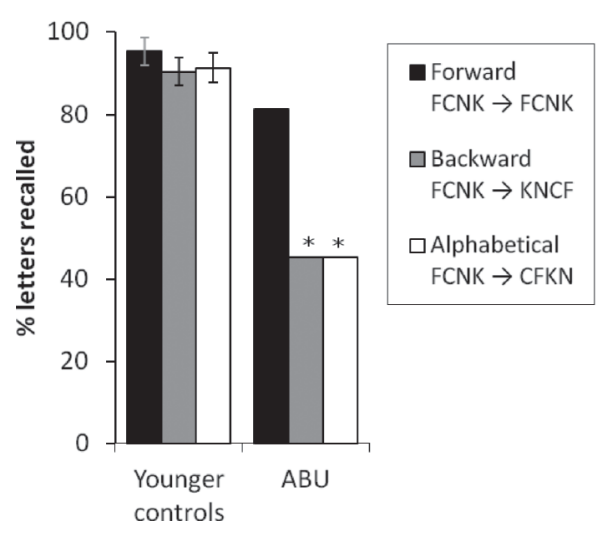

Figure 5. Letter recall task. Asterisks denote significantly impaired performance (modified $t$-test; $p<.05$ ).

Evidence for impairment in the patients. Accuracy for forward, backward, and alphabetical recall of five-letter lists is shown in Figure 5A. The timing of the cue had little effect on performance so results were averaged over pre- and post-presentation cues. RSDT was used to test for dissociations between particular conditions, relative to controls. Patient JB showed a selective deficit for recall in alphabetical order. He showed no dissociation between forward and backward recall $\left(t_{1}=0.68, p=.25 ; t_{2}=\right.$ $0.70, p=.25)$ but did display a larger difference than controls for forward vs alphabetical $\left(t_{1}=3.90, p<.001 ; t_{2}=3.47, p=.005\right)$ and backward vs alphabetical recall $\left(t_{1}=3.81, p<.001 ; t_{2}=2.67, p=.016\right)$. In contrast, JHU showed preserved forward recall but was impaired at backward and alphabetical recall. He showed an abnormal dissociation between both forward and backward $\left(t_{1}=4.07, p<.001 ; t_{2}=3.81\right.$, $p=.004)$ and forward and alphabetical memory $\left(t_{1}=5.26, p<.001 ; t_{2}=4.28, p=\right.$ $.003)$. He also exhibited a dissociation between backward and alphabetical recall, but only when compared to his age-matched subgroup $\left(t_{1}=1.24, p=.12 ; t_{2}=4.12, p=\right.$ $.003)$. This suggests that his alphabetical recall was even more severely impaired than his backward recall. 
Finally, ABU performed worst of the three patients and was significantly impaired in all three conditions. He did not show any significant dissociations between conditions. However, because he performed poorly on forwards recall we also tested him with easier four-letter lists (see Figure 5B). On these lists his performance was more similar to that of JHU: forward recall was not impaired, but backward and alphabetical recall were. There was a significant dissociation for forward vs. backward $(t=4.59$, $p=.002)$ and forward vs. alphabetical $(t=2.60, p=.02)$ but no effect for backward vs alphabetical $(t=0.91, p=.20)$.

On the forced-choice alphabetical knowledge test, JB and JHU both scored 28/28, indicating that their knowledge of the alphabet was intact. ABU was somewhat less successful, scoring only 24/28. This could explain why he did poorly on the alphabetical recall condition.

\section{Discussion}

This experiment produced further evidence for a highly specific executive control deficit in patient JB. He performed well when reversing the order of letter strings, indicating preserved executive function when required to manipulate the contents of working memory. However, he was not able to perform order manipulations based on his stored knowledge of the alphabet. This dissociation cannot be explained in terms of task difficulty because controls performed equally well in alphabetical and backwards conditions. Nor was it a result of impaired knowledge of the alphabetical order of letters. Instead, JB appeared to have specific difficulty using his intact alphabetical knowledge to direct his responses. This is similar to the semantic control deficits observed in this patient previously, in which he appeared to have intact semantic knowledge but difficulty controlling retrieval of this knowledge such that it was appropriate to the current task or context. In contrast, ABU and JHU were equally impaired at backwards and alphabetical recall, suggesting they had a more general executive deficit, affecting their ability to perform any kind of online manipulation of the contents of working memory.

\section{GENERAL DISCUSSION}

The ability to access semantic knowledge in a flexible and context-appropriate way, referred to here as "semantic control," is a critical aspect of semantic cognition. Neuroimaging evidence indicates that the neural substrate of semantic control at least partially overlaps with that of executive control in other domains, suggesting that a common system might govern all forms of cognitive control (Duncan, 2010; Duncan \& Owen, 2000; Jefferies \& Lambon Ralph, 2006). In line with this view, in two cases with relatively "pure" semantic control deficits we found evidence that non-semantic aspects of executive processing were also impaired. However, in a third patient, JB, there was a clear dissociation between semantic and non-semantic control. This individual was able to perform the key executive functions of updating and manipulation of information in working memory, as well as dividing attention between two tasks, provided that these operations did not involve semantic knowledge. Conversely his ability to perform controlled processing on semantic information was impaired. This suggests that semantic control depends at least partially on unique resources not required for other forms of executive processing. 
Before turning to the theoretical implications of these results we will consider some alternative explanations for why JB should show a different profile to the other two patients. JB performs slightly better than ABU and JHU on semantic tasks in general (see Table 1), so one possibility is that the observed differences are a result of severity. It could be that all three patients have a domain-general executive impairment but that, because JB's deficit is the mildest, we have failed to detect it in this study. This explanation would need to take into account the fact that in our previous study, JB did show significant impairment on a number of executively demanding semantic judgements (Hoffman, Jefferies, et al., 2011). However, it could be that the tasks in the present study are less demanding than those we used previously to assess semantic control, and therefore less sensitive to mild impairments. This is unlikely because the tasks in the present study were designed to be challenging even for healthy participants; indeed, in each experiment the control group only achieved around $80 \%$ accuracy in the most difficult condition. Thus, while one would expect even the mildest impairment to disrupt performance on these tasks, no deficits were detected in JB. In contrast, in our previous study of semantic control, healthy participants performed close to ceiling in each experiment (typically above $95 \%$ accurate) yet JB was impaired on these easier tasks. We are confident, therefore, that JB's profile is not a result of differential task sensitivity.

Another possibility is that JB has a mild deficit in semantic knowledge that prevents him from performing normally on semantic tasks. This theory is supported by the extent of his lesion which, in addition to affecting an area of the left pMTG associated with semantic control (discussed below), extends rostrally to include the anterior fusiform. Bilateral damage to this area is associated with the progressive degradation of semantic knowledge in semantic dementia (Mion et al., 2010) and unilateral lesions can sometimes result in mild impairments (Lambon Ralph, Cipolotti, Manes, \& Patterson, 2010). Perhaps JB's semantic knowledge store has been subtly disrupted, affecting only the most demanding semantic tasks. In Experiment 4 we investigated the issue of knowledge storage vs controlled regulation. JB showed fully intact knowledge of the alphabet but was still drastically impaired in the alphabetical recall condition. This suggests that his deficit on this task stemmed not from loss of knowledge but from an inability to use his knowledge to regulate behaviour.

The dissociation between semantic and non-semantic control in patient JB suggests that at least some aspects of the neural and cognitive architecture underpinning executive control are specialised for processing semantic knowledge. This is perhaps not surprising, given that semantic memory comprises a vast and rich store of information that is central to our interaction with the environment. It does not necessarily imply that the neural underpinnings of semantic control are entirely independent of those for executive processing in other domains. Indeed, a large body of neuroimaging evidence suggests that regions of prefrontal and inferior parietal cortex are reliably activated for executively demanding tasks across a range of processing domains (Collette et al., 2006; Duncan, 2006; Duncan \& Owen, 2000; Nee et al., 2007). Duncan and colleagues refer to these as "multiple demand" regions and suggest that they are recruited whenever task-dependent controlled processing is required, irrespective of the task involved (Duncan, 2010). Neuroimaging studies of semantic control reveal similar activations, most consistently in the inferior frontal gyrus but also in inferior parietal cortex (Badre et al., 2005; Rodd et al., 2005; Thompson-Schill et al., 1997; Wagner et al., 2001), suggesting that "multiple demand" regions are also involved in controlled semantic processing. 
In addition to this shared neural substrate some regions have been selectively associated with controlled processing in semantic tasks. Posterior middle temporal gyrus (pMTG) is often activated in manipulations of high vs low semantic control (Bedny et al., 2008; Gold et al., 2006; Noppeney et al., 2004; Rodd et al., 2005; Whitney, Jefferies, et al., 2011) and TMS applied to this region slows executively demanding semantic judgements, with no commensurate effect on executively demanding perceptual decisions (Whitney, Kirk, et al., 2011). This region is not implicated in the "multiple demand" system. Likewise, the anterior portion of inferior prefrontal cortex (BA45/47) responds strongly to manipulations of semantic control but not to executively demanding tasks in other domains (Gold \& Buckner, 2002; Gough et al., 2005; Nagel et al., 2008; Snyder et al., 2007). Two recent TMS studies have also shown that stimulation of this region slows executively demanding semantic decisions with no effect on matched visual or numerical tasks (Hoffman, Jefferies, \& Lambon Ralph, 2010; Whitney, Kirk, et al., 2011). A recent formal meta-analysis of semantic control studies supports these findings (Noonan, Jefferies, Visser, \& Lambon Ralph, 2012). While a number of regions were found to be consistently activated in high vs low semantic control contrasts across studies, only anterior, inferior prefrontal cortex and pMTG were more active for semantic control than for non-semantic executive control.

The most likely cause of the semantic-specific control deficit in patient JB is damage to pMTG, as his lesion does not affect prefrontal or parietal cortex (see Figure 1). It is more difficult to ascertain the cause of the more general executive deficits in patients $\mathrm{ABU}$ and $\mathrm{JHU}$, as high-resolution imaging was not available for these patients, but it seems possible that damage affects one or more "multiple demand" regions required for domain-general controlled processing. The main contribution of this study, however, is not to provide precise anatomical localisation of the semantic control network. Instead this study, along with the TMS studies discussed earlier, indicates that with selective damage to elements of the semantic control network, a behavioural dissociation between semantic and non-semantic control can be observed. This confirms some regions of cortex are critical for control and regulation of semantic knowledge but are not involved in other forms of controlled processing. Future studies should clarify the precise functions of semantic-specific control regions vs domaingeneral "multiple demand" regions, since it is likely that interaction between the various components of this distributed network is necessary to give rise to appropriate, semantically driven behaviour.

Manuscript received 25 January 2012
Manuscript accepted 18 November 2012
First published online 1 March 2013

\section{REFERENCES}

Awh, E., Jonides, J., Smith, E. E., Schumacher, E. H., Koeppe, R. A., \& Katz, S. (1996). Dissociation of storage and rehearsal in verbal working memory: Evidence from positron emission tomography. Psychological Science, 7, 25-31.

Baayen, R. H., Piepenbrock, R., \& van Rijn, H. (1993). The CELEX lexical database (CD-ROM). Philadelphia: Linguistic Data Consortium, University of Pennsylvania.

Baddeley, A. (1996). Exploring the central executive. Quarterly Journal of Experimental Psychology Section a-Human Experimental Psychology, 49, 5-28.

Badre, D., Poldrack, R. A., Pare-Blagoev, E. J., Insler, R. Z., \& Wagner, A. D. (2005). Dissociable controlled retrieval and generalized selection mechanisms in ventrolateral prefrontal cortex. Neuron, 47, 907-918. 
Badre, D., \& Wagner, A. D. (2002). Semantic retrieval, mnemonic control, and prefrontal cortex. Behavioral and Cognitive Neuroscience Reviews, 1, 206-218.

Bedny, M., McGill, M., \& Thompson-Schill, S. L. (2008). Semantic adaptation and competition during word comprehension. Cerebral Cortex, 18, 2574-2585.

Belleville, S., Rouleau, N., \& Caza, N. (1998). Effect of normal aging on the manipulation of information in working memory. Memory \& Cognition, 26, 572-583.

Bozeat, S., Lambon Ralph, M. A., Patterson, K., Garrard, P., \& Hodges, J. R. (2000). Non-verbal semantic impairment in semantic dementia. Neuropsychologia, 38, 1207-1215.

Bullard, S. E., Fein, D., Gleeson, M. K., Tischer, N., Mapou, R. L., \& Kaplan, E. (2004). The Biber Cognitive Estimation Test. Archives of Clinical Neuropsychology, 19, 835-846.

Burgess, P., \& Shallice, T. (1997). The Hayling and Brixton Tests. Suffolk: Thames Valley Test Company.

Collette, F., Hogge, M., Salmon, E., \& Van der Linden, M. (2006). Exploration of the neural substrates of executive functioning by neuroimaging. Neuroscience, 139, 209-221.

Collette, F., Salmon, E., Van der Linden, M., Chicherio, C., Belleville, S., Degueldre, C., ... Franck, G. (1999). Regional brain activity during tasks devoted to the central executive of working memory. Cognitive Brain Research, 7, 411-417.

Corbett, F., Jefferies, E., \& Lambon Ralph, M. A. (2009). Exploring multimodal semantic control impairments in semantic aphasia: Evidence from naturalistic object use. Neuropsychologia, 47, 2721-2731.

Corbett, F., Jefferies, E., \& Lambon Ralph, M. A. (2011). Deregulated semantic cognition follows prefrontal and temporoparietal damage: Evidence from the impact of task constraint on non-verbal object use. Journal of Cognitive Neuroscience, 23, 1125-1135.

Crawford, J. R., \& Garthwaite, P. H. (2005). Testing for suspected impairments and dissociations in singlecase studies in neuropsychology: Evaluation of alternatives using Monte Carlo simulations and revised tests for dissociations. Neuropsychology, 19, 318-331.

Crawford, J. R., \& Howell, D. C. (1998). Comparing an individual's test score against norms derived from small samples. The Clinical Neuropsychologist, 12, 482-486.

Cristescu, T. C., Devlin, J. T., \& Nobre, A. C. (2006). Orienting attention to semantic categories. Neuroimage, 33, 1178-1187.

Davis, C. J. (2005). N-Watch: A program for deriving neighborhood size and other psycholinguistic statistics. Behavior Research Methods, 37, 65-70.

Dosenbach, N. U. F., Fair, D. A., Cohen, A. L., Schlagger, B. L., \& Petersen, S. E. (2008). A dual-networks architecture of top-down control. Trends in Cognitive Sciences, 12, 99-105.

Duncan, J. (2006). Brain mechanisms of attention. Quarterly Journal of Experimental Psychology, 59, 2-27.

Duncan, J. (2010). The multiple-demand (MD) system of the primate brain: Mental programs for intelligent behaviour. Trends in Cognitive Sciences, 14, 172-179.

Duncan, J., \& Owen, A. M. (2000). Common regions of the human frontal lobe recruited by diverse cognitive demands. Trends in Neurosciences, 23, 475-483.

Engle, R. W. (2002). Working memory capacity as executive attention. Current Directions in Psychological Science, 11, 19-23.

Engle, R. W., Tuholski, S. W., Laughlin, J. E., \& Conway, A. R. A. (1999). Working memory, short-term memory, and general fluid intelligence: A latent-variable approach. Journal of Experimental Psychology: General, 128, 309-331.

Gold, B. T., Balota, D. A., Jones, S. J., Powell, D. K., Smith, C. D., \& Andersen, A. H. (2006). Dissociation of automatic and strategic lexical-semantics: Functional magnetic resonance imaging evidence fro differing roles of multiple frontotemporal regions. Journal of Neuroscience, 26, 6523-6532.

Gold, B. T., \& Buckner, R. L. (2002). Common prefrontal regions coactivate with dissociable posterior regions during controlled semantic and phonological tasks. Neuron, 35, 803-812.

Gough, P. M., Nobre, A. C., \& Devlin, J. T. (2005). Dissociating linguistic processes in the left inferior frontal cortex with transcranial magnetic stimulation. Journal of Neuroscience, 25, 8010-8016.

Hoffman, P., Jefferies, E., Ehsan, S., Hopper, S., \& Lambon Ralph, M. A. (2009). Selective short-term memory deficits arise from impaired domain-general semantic control mechanisms. Journal of Experimental Psychology: Learning, Memory and Cognition, 35, 137-156.

Hoffman, P., Jefferies, E., \& Lambon Ralph, M. A. (2010). Ventrolateral prefrontal cortex plays an executive regulation role in comprehension of abstract words: Convergent neuropsychological and rTMS evidence. Journal of Neuroscience, 46, 15450-15456.

Hoffman, P., Jefferies, E., \& Lambon Ralph, M. A. (2011). Explaining semantic short-term memory deficits: Evidence for the critical role of semantic control. Neuropsychologia, 49, 368-381. 
Hoffman, P., Rogers, T. T., \& Lambon Ralph, M. A. (2011). Semantic diversity accounts for the "missing" word frequency effect in stroke aphasia: Insights using a novel method to quantify contextual variability in meaning. Journal of Cognitive Neuroscience, 23, 2432-2446.

Jefferies, E., Hoffman, P., Jones, R., \& Lambon Ralph, M. A. (2008). The impact of semantic impairment on verbal short-term memory in stroke aphasia and semantic dementia: A comparative study. Journal of Memory and Language, 58, 66-87.

Jefferies, E., \& Lambon Ralph, M. A. (2006). Semantic impairment in stroke aphasia vs. semantic dementia: A case-series comparison. Brain, 129, 2132-2147.

Jefferies, E., Patterson, K., \& Lambon Ralph, M. A. (2008). Deficits of knowledge versus executive control in semantic cognition: Insights from cued naming. Neuropsychologia, 46, 649-658.

Kan, I. P., \& Thompson-Schill, S. L. (2004). Effect of name agreement on prefrontal activity during overt and covert picture naming. Cognitive, Affective and Behavioral Neuroscience, 4, 43-57.

Koenig, K., \& Grossman, M. (2007). Process and content in semantic memory. In J. Hart \& M. A. Kraut (Eds.), Neural basis of semantic memory (pp. 247-264). Cambridge: Cambridge University Press.

Lambon Ralph, M. A., Cipolotti, L., Manes, F., \& Patterson, K. (2010). Taking both sides: Do unilateral anterior temporal lobe lesions affect semantic memory? Brain, 133, 3243-3255.

Mahon, B. Z., \& Caramazza, A. (2009). Concepts and categories: A cognitive neuropsychological perspective. Annual Review of Psychology, 60, 27-51.

Martin, A. J. (2007). The representation of object concepts in the brain. Annual Review of Psychology, 58, $25-45$.

Martin, R. C., Shelton, J., \& Yaffee, L. S. (1994). Language processing and working memory: Neuropsychological evidence for separate phonological and semantic capacities. Journal of Memory and Language, 33, 83-111.

Mion, M., Patterson, K., Acosta-Cabronero, J., Pengas, G., Izquierdo-Garcia, D., Hong, Y. T., Nestor, P. J. (2010). What the left and right fusiform gyri tell us about semantic memory. Brain, 133, 3256-3268.

Miyake, A., Friedman, N. P., Emerson, M. J., Witzki, A. H., Howerter, A., \& Wager, T. D. (2000). The unity and diversity of executive functions and their contributions to complex "frontal lobe" tasks: A latent variable analysis. Cognitive Psychology, 41, 49-100.

Nagel, I. E., Schumacher, E. H., Goebel, R., \& D’Esposito, M. (2008). Functional MRI investigation of verbal selection mechanisms in lateral prefrontal cortex. Neuroimage, 43, 801-807.

Nee, D. E., Wager, T. D., \& Jonides, J. (2007). Interference resolution: Insights from a meta-analysis of neuroimaging tasks. Cognitive Affective \& Behavioral Neuroscience, 7(1), 1-17.

Noonan, K. A., Jefferies, E., Corbett, F., \& Lambon Ralph, M. A. (2010). Elucidating the nature of deregulated semantic cognition in semantic aphasia: Evidence for the roles of the prefrontal and temporoparietal cortices. Journal of Cognitive Neuroscience, 22, 1597-1613.

Noonan, K. A., Jefferies, E., Visser, M., \& Lambon Ralph, M. A. (2012). Aligning evidence from functional neuroimaging and neuropsychology for the neural network underpinning semantic control: A meta-analytic investigation. Manuscript submitted for publication.

Noppeney, U., Phillips, J., \& Price, C. (2004). The neural areas that control the retrieval and selection of semantics. Neuropsychologia, 42, 1269-1280.

Patterson, K., Nestor, P. J., \& Rogers, T. T. (2007). Where do you know what you know? The representation of semantic knowledge in the human brain. Nature Reviews Neuroscience, 8, 976-987.

Peers, P. V., Ludwig, C. J. H., Rorden, C., Cusack, R., Bonfiglioli, C., Bundesen, C., . . Duncan, J. (2005). Attentional functions of parietal and frontal cortex. Cerebral Cortex, 15, 1469-1484.

Raven, J. C. (1992). Standard progressive matrices: 1992 Edition. Windsor: NFER-Nelson.

Robertson, I. H., Ward, T., Ridgeway, V., \& Nimmo-Smith, I. (1994). The test of everyday attention. Flempton: Thames Valley Test Company.

Rodd, J. M., Davis, M. H., \& Johnsrude, I. S. (2005). The neural mechanisms of speech comprehension: fMRI studies of semantic ambiguity. Cerebral Cortex, 15, 1261-1269.

Saffran, E. M. (2000). The organization of semantic memory: In support of a distributed model. Brain and Language, 71, 204-212.

Saffran, E. M., Bogyo, L., Schwartz, M. F., \& Marin, O. S. M. (1980). Does deep dyslexia reflect right hemisphere reading? In M. Coltheart, J. C. Marshall, \& K. E. Patterson (Eds.), Deep dyslexia (pp. 361-406). London: Routledge \& Kegan Paul.

Samson, D., Connolly, C., \& Humphreys, G. W. (2007). When "happy" means "sad": Neuropsychological evidence for the right prefrontal cortex contribution to executive semantic processing. Neuropsychologia, 45, 896-904. 
Snyder, H. R., Feigenson, K., \& Thompson-Schill, S. L. (2007). Prefrontal cortical response to conflict during semantic and phonological tasks. Journal of Cognitive Neuroscience, 19, 761-775.

Soni, M., Lambon Ralph, M. A., Noonan, K., Ehsan, S., Hodgson, C., \& Woollams, A. M. (2009). "L" is for tiger: Effects of phonological (mis)cueing on picture naming in semantic aphasia. Journal of Neurolinguistics, 22, 538-547.

Stuss, D. T., Levine, B., Alexander, M. P., Hong, J., Palumbo, C., Hamer, L., . . Izukawa, D. (2000). Wisconsin Card Sorting Test performance in patients with focal frontal and posterior brain damage: Effects of lesion location and test structure on seperable cognitive processes. Neuropsychologia, 38, $388-402$.

Thompson-Schill, S. L., D'Esposito, M., Aguirre, G. K., \& Farah, M. J. (1997). Role of left inferior prefrontal cortex in retrieval of semantic knowledge: A reevaluation. Proceedings of the National Academy of Sciences of the United States of America, 94, 14792-14797.

Tombaugh, T. N. (2004). Trail making Test A and B: Normative data stratified by age and education. Archives of Clinical Neuropsychology, 19, 203-214.

Treiman, R., \& Danis, C. (1988). Short-term memory errors for spoken syllables are affected by the linguistic structure of the syllables. Journal of Experimental Psychology: Learning, Memory and Cognition, 14, 145-152.

Treiman, R., \& Kessler, B. (1995). In defense of an onset-rime syllable structure for English. Language and Speech, 38, 127-142.

Unsworth, N., Heitz, R. R., Schrock, J. C., \& Engle, R. W. (2005). An automated version of the operation span task. Behavior Research Methods, 37, 498-505.

Wagner, A. D., Pare-Blagoev, E. J., Clark, J., \& Poldrack, R. A. (2001). Recovering meaning: Left prefrontal cortex guides controlled semantic retrieval. Neuron, 31, 329-338.

Warrington, E. K., \& James, M. (1991). The Visual Object and Space Perception Battery. Bury St. Edmunds: Thames Valley Test Company.

Whitney, C., Jefferies, E., \& Kircher, T. (2011). Heterogeneity of the left temporal lobe in semantic representation and control: Priming multiple vs. single meanings of ambiguous words. Cerebral Cortex, 21, 831-844.

Whitney, C., Kirk, M., O’Sullivan, J., Lambon Ralph, M. A., \& Jefferies, E. (2011). The neural organization of semantic control: TMS evidence for a distributed network in left inferior frontal and posterior middle temporal gyrus. Cerebral Cortex, 21, 1066-1075.

Whitney, C., Kirk, M., O’Sullivan, J., Lambon Ralph, M. A., \& Jefferies, E. (2012). Executive-semantic processing is underpinned by a large-scale neural network: Revealing the contribution of left prefrontal, posterior temporal and parietal cortex to controlled retrieval and selection using TMS. Journal of Cognitive Neuroscience, 24, 133-147.

Yarkoni, T., Balota, D., \& Yap, M. (2008). Moving beyond Coltheart's N: A new measure of orthographic similarity. Psychonomic Bulletin \& Review, 15, 971-979.

Ziegler, J. C., \& Goswami, U. (2005). Reading acquisition, developmental dyslexia, and skilled reading across languages: A psycholinguistic grain size theory. Psychological Bulletin, 131, 3-29. 